\title{
A health economic simulation model for the clinical management of osteoporosis.
}

E Jonsson, A Hansson-Hedblom, Ö Ljunggren, K Åkesson, Anna Spångeus, J A Kanis and F Borgström

The self-archived postprint version of this journal article is available at Linköping University Institutional Repository (DiVA):

http://urn.kb.se/resolve?urn=urn:nbn:se:liu:diva-144030

N.B.: When citing this work, cite the original publication.

The original publication is available at www.springerlink.com:

Jonsson, E, Hansson-Hedblom, A, Ljunggren, Ö, Ảkesson, K, Spångeus, A., Kanis, J A, Borgström, F, (2017), A health economic simulation model for the clinical management of osteoporosis., Osteoporosis International.

https://doi.org/10.1007/s00198-017-4325-4

Original publication available at:

https://doi.org/10.1007/s00198-017-4325-4

Copyright: Springer Verlag (Germany) (Osteoporosis International)

http://www.springerlink.com/?MUD=MP 


\section{A Health Economic Simulation Model for the Clinical Management of Osteoporosis}

Authors: Emma Jonsson ${ }^{1}$, Amanda Hansson-Hedblom ${ }^{1}$, Östen Ljunggren ${ }^{2}$, Kristina Åkesson ${ }^{3}$, Anna Spångeus ${ }^{4}$, John A Kanis ${ }^{5,6}$, Fredrik Borgström ${ }^{1,7}$

1. Quantify Research, Stockholm, Sweden

2. Department of Medical Sciences, Uppsala University Hospital, Uppsala, Sweden

3. Lund University, Dept of Clinical Sciences, Clinical and Molecular Osteoporosis Unit, Malmö, Sweden

4. Department of Endocrinolog Dept Medicine and Health, Linköping University Hospital, Linköping, Sweden

5. University of Sheffield, Sheffield, UK

6. Catholic University of Australia, Melbourne, Australia

7. LIME/MMC, Karolinska Institutet, Stockholm, Sweden

Corresponding Author:

(凶) Emma Jonsson

Quantify Research

Hantverkargatan 8

SE-112 21 Stockholm, Sweden

Phone: +46 739477189

E-mail: emma.jonsson@quantifyresearch.com

Research ethics committee: Stockholm ethics vetting committee decision 2013/1543-31/4

Acknowledgements: The authors gratefully acknowledge the guidance of the Epidemiology and Quality of Life Working Group of the Committee of Scientific Advisors of the International Osteoporosis Foundation. The study was financed with an unrestricted grant from Medtronic. 
Conflict of interest: EJ, AH and FB have previously consulted for companies marketing products for osteoporosis. ÖJ, AS, KÅ, JK declare that they have no conflict of interest.

\section{Mini-abstract}

The objective was to estimate the burden of osteoporosis in Sweden based on current clinical practice and the costeffectiveness of improvements in the management of osteoporosis over the clinical management compared to current clinical practice. Results showed that better compliance to treatment guidelines is associated with better projected outcomes and cost-savings. 


\section{Abstract}

\section{Purpose}

To estimate the burden of osteoporosis in Sweden based on current clinical practice and the cost-effectiveness of improvements in the management of osteoporosis over the clinical management compared to current clinical practice.

\section{Methods}

The analysis was carried out using a model that simulates the individual patients considered for pharmacological treatment during one year and their projected osteoporosis treatment pathway, quality adjusted life years (QALYs) and costs over their remaining lifetime. All patients regardless of treatment or no treatment were simulated. Information on current management of osteoporosis in terms of patient characteristics and treatment patterns were derived from a Swedish osteoporosis research database based on national registers and patient records. Current (standard) clinical management was compared with alternative scenarios mirroring Swedish treatment guidelines.

\section{Results}

The national burden in terms of lost QALYs was estimated at 14,993 QALYs and the total economic cost at €776M. Scenario analyses showed that 382-3,864 QALYs could be gained at a cost/QALY ranging from cost-saving to $€ 31,368$, depending on the scenario. The margin of investment, i.e. the maximum amount that could be invested in the healthcare system to achieve these improvements up to the limit of the willingness to pay/QALY, was estimated at $€ 199 \mathrm{M}$ on a population level (€3,634/patient).

\section{Conclusions}

The analysis showed that better compliance to treatment guidelines is associated with better projected outcomes and cost-savings. From a cost-effectiveness perspective, there is also considerable room for investment to achieve these improvements in the management of osteoporosis. 


\section{Keywords}

Osteoporosis, Fracture, Cost, Quality-of-life, Sweden, Register

\section{Introduction}

It has been estimated that osteoporosis causes 9 million fractures annually worldwide and the frequency is rising in many countries [1]. Osteoporotic fractures are associated with major societal costs. For example, the total monetary burden of osteoporosis including fracture associated costs as well as pharmacological interventions in 27 EU countries was estimated at €37,378 million in 2010 [2]. In addition, osteoporotic fractures account for 2 million disabilityadjusted life years lost annually in Europe [3].

Most previous health economic analyses in osteoporosis have evaluated the cost-effectiveness for an osteoporosis drug in specified patient populations [4, 5]. For example, the cost-effectiveness of treating a 70-year old woman for 5 years with bisphosphonates compared to no treatment. Although most available treatments for fracture prevention have been shown to be cost-effective in patients with increased fracture risk in varying patient groups, there is an apparent gap between treatment eligibility according to criteria stipulated in guidelines compared with the actual clinical practice. This “treatment gap" has been estimated at 23-80\% depending on sex and age group in Sweden [6]. Similar estimates have been found in the UK, Germany, US and Spain [3, 7]. In addition to this treatment gap, in those who start treatment, persistence with osteoporosis treatments is poor in clinical practice-approximately $50 \%$ of patients discontinue after one year-which is shown to reduce anti-fracture efficacy of treatment [8]. In tandem, few patients switch to another treatment after discontinuation [6]. The future burden of osteoporosis, which is anticipated to grow due to aging populations, could potentially be lowered by reducing this treatment gap. Whether osteoporosis is treated cost-effectively based on current treatment patterns and uptake in clinical practice has not been thoroughly evaluated.

It is of relevance to understand the current clinical management of osteoporosis for several reasons. Better understanding of the current clinical management would inform whether current osteoporosis medications are being used in accordance with guidelines. By constructing a simulation model that can synergize real world data and other existing data, it would be possible to assess the costs and consequences over the treatment pathway. Also, such a model 
could inform how changes in current clinical management, such as closing the treatment gap, could improve the outcomes for the patient population and reduce the burden of disease on a population level. A model that could accommodate all these types of analyses would be very informative for payers, providers, researchers, and would ultimately benefit the patient.

The objective of this study was to develop a simulation model that reflects the osteoporosis clinical management at the population level and to demonstrate its functionality by estimating the burden of disease and the potential costeffectiveness by changes in the management of osteoporosis across the clinical management compared to current clinical practice in Sweden.

\section{Material and Methods}

\section{Model Design}

A health economic microsimulation model was developed, aiming to simulate a cohort representative of a real-world osteoporosis patient population. In the model, patients transition between health states and costs and consequences are tracked over the clinical management. A patient starts the model at clinical attention where he/she is being considered for an osteoporosis treatment (henceforth “index-event”) (Fig. 1). From this point, the patient moves through a decision tree. For the current analyses, the decision tree consists of the following events; having/not having BMD measurement, and starting/not starting osteoporosis treatments. Patients can start treatment without preceding BMD measurement.

Events in the decision tree are structured in separate branches and are handled as instantaneous, meaning that no time is assumed to elapse from the first event in the decision tree until the last event in the tree. When a treatment decision is made, the patient enters a Markov model in which he/she remains for the rest of the model simulation. The Markov model consists of six health states whose transition probabilities are similar to previous osteoporosis models [5, 9, 10]. All patients start in the Baseline state, and in each following cycle, the patient is at risk of sustaining a fracture at the hip, clinical vertebrae, wrist or “other" site, or die. Fractures were characterised as osteoporotic when they were associated with low bone mass and their incidences increases after the age of 50. "Other” fracture is a composite of fractures including a wider range of osteoporotic fractures (humerus, clavicle, rib, tibia, pelvis, fibula, scapula, sternum, 
and other femoral fractures). Patients transition back to the baseline state if no fracture or death occurs. Hip and vertebral fractures are assumed to incur long-term costs and quality of life (QoL) decrements. These long-term effects are not modelled as separate post-fracture health states, in contrast to many previous models in osteoporosis, but are incorporated by “tracking” patient history.

The cycle length was 12 months and patients are tracked from treatment consideration until death or age of 100 years.

[Figure 1]

The model was designed to include only pharmacological intervention treatments. The time a patient remains on treatment is directly associated with the duration of efficacy. Osteoporosis models commonly assume a maximum 5year treatment duration, during which patients are at risk of becoming non-persistent [5, 9]. The current model was built to accommodate more flexible treatment patterns reflecting that in reality, patients may switch, drop out and restart treatment over time. The model therefore considers that patients who do not start treatment at the index-event have time-dependent probabilities of starting treatment from index-event and for the rest of the model simulation. Further, the model allows for non-persistent patients to switch to next line of treatment. The events of non-persistence, starting and switching treatment are allowed during the entirety of the model simulation and are handled as instantaneous events.

There is a consensus that anti-fracture efficacy remains after ending treatment for a period of time ("offset time”). Offset time can be modelled with two different assumptions; 1) offset time is assumed to correspond to the time on treatment (consequently, offset time is shorter for patients who drop out earlier during the intended treatment duration), or 2) all patients have the same specified offset time irrespective of whether a patient drops out earlier than the intended duration. The latter approach may be reasonable if patients stay on treatment for several years, however including discontinuation explicitly like in this analysis, a 5-year offset would give 5 years of "free" effect to a patient with a 1year treatment. In the current analyses, the offset time was therefore assumed to be equal to the time on treatment, thus patients with shorter treatment lengths have shorter offset time. 


\section{Data}

\section{Patient population}

The target population in the current analysis corresponds to Swedish men and women $\geq 50$ years that during one year would be subject to osteoporosis treatment consideration due to typical osteoporotic fracture, secondary osteoporosis due to glucocorticoid use, and being at high risk due to other reasons.

\section{Fracture risk}

Health economic models for osteoporosis are generally populated with fracture risk in the general population, adjusted for clinical risk factors (CRFs) of the patient population targeted in the analysis and the effect of treatment. The FRAX ${ }^{\circledR}$ algorithm considers multiple risk factors (age, T-score, previous fracture, glucocorticoid use, other secondary osteoporosis, rheumatoid arthritis) and its use is intended for identifying patients eligible for treatment based on fracture probability. The FRAX tool was included in the model to facilitate fracture risk estimation for patients with different sets of risk factors. The prevalence of CRFs, and the correlation between CRFs, in the target patient population was derived from FRAX cohorts. Given a patient's CRFs, the relative risk of fracture compared to normal population risk were derived using the FRAX algorithm at the beginning of the model simulation. The occurrence of fractures along the model simulation is anticipated to increase the risk of subsequent fracture. The relative risk was therefore updated every fifth year in the model, by updating the fracture history and age. Other CRFs were assumed constant, since there is a lack of data on whether the presence of those CRFs changes over time.

The risk of fractures at the hip, clinical vertebrae, wrist and other sites in the general Swedish population were derived from a population-based study in Malmö [11, 12].

\section{Mortality}

Age and gender specific mortality rates for the general population were based on Swedish national statistics [13]. Hip and vertebral fractures were assumed to cause long-term increased mortality, while other fractures were assumed to cause increased mortality only in the year following fracture, and wrist fractures were not assumed to cause increased mortality. Relative risks of death in patients with fracture compared with the general population were derived from Ström et al. [3, 5, 14]. Patients with osteoporosis have a higher degree of frailty compared with the general population 
[15-17]. Therefore, we assumed that $30 \%$ of the relative risks were associated with the event in accordance with previous economic studies. Increased mortality was assumed to remain for eight years, which is the follow-up duration in studies of excess post-fracture mortality $[15,17,18]$.

\section{Costs}

Costs (€EUR 2016) were included from a healthcare perspective thus considering only direct costs. In accordance with Swedish guidelines, costs and effects were discounted at 3\% annually [19]. Direct medical costs for fractures were derived from Swedish studies [5, 20]. Daily cost of residing at nursing home was retrieved from a previous costeffectiveness study [5].

Costs per package of osteoporosis drugs were based on the most recent prices from the Swedish Dental and Pharmaceutical Benefits Agency [21]. Treatment monitoring was assumed to include a yearly physician visit (unit cost: €175) and DXA measurement every second year (unit cost: €160) [22]. For intravenous/subcutaneous drugs, an administration unit cost of $€ 127$ was included. Patients were assumed to be monitored after treatment discontinuation, including a DXA measurement every fifth year and a physician visit every second year. Unit costs were sourced from Swedish regional price lists [22].

Costs were inflated to 2016 using consumer price index [23] where needed and converted to EUR using the average 2016 exchange rate (1 EUR=9.47 SEK [24]).

\section{Quality of life}

All fracture types were assumed to impact QoL in the first year after a fracture. Hip and vertebral fractures were also assumed to impact QoL in the second and subsequent years. Fracture-related QoL multipliers were primarily based on the ICUROS multi-country study collecting EQ-5D 18 months after hip, vertebral and distal forearm fractures [25]. QoL multiplier for other fractures was collected from a Swedish study [26]. These were multiplied with age and gender specific QoL tariffs for the general population based on Swedish EQ-5D estimates [27]. 


\section{Treatment patterns in the current clinical management}

A database consisting of patient-level register data from Swedish national registers (the Swedish Osteoporosis Research Database, SWORD) was used to inform on current clinical managements. SWORD included Swedish patients with a primary diagnosis of a fragility fracture after the age of 50, initiation of osteoporosis pharmacological treatment, and/or a DXA scan performed at one of the participating osteoporosis clinic in Uppsala (Akademiska sjukhuset), Linköping (Universitetssjukhuset i Linköping) or Malmö (Skåne Universitetssjukhus) between 2006-0601 and 2012-12-31. From the data, it was not possible to determine if a fracture was caused by low energy trauma (fragility fracture) [12]. The inclusion criteria for the database therefore defined a fragility fracture as a fracture type commonly associated with osteoporosis. The included fracture types and corresponding ICD-10 codes were: rib, sternum, thoracic spine (ICD-10: S22.x), lumbar spine pelvis (ICD-10: S32.x), shoulder (ICD-10: S42.x), forearm (ICD-10: S52.x), wrist and hand (ICD-10: S62.0x, S62.8), femur including hip (ICD-10: S72.x), lower leg (ICD-10: S82.x), spine unspecified (ICD-10: T08.x), upper limb unspecified (ICD-10: T10.x), lower limb unspecified (ICD-10: T12.x), and osteoporosis with pathological fracture (ICD-10: M80.x).

The target patient population for the model was stratified in six cohorts; fracture at the hip, clinical vertebrae, wrist or other site, secondary osteoporosis due to glucocorticoid use and other high risk. The cohorts were identified in SWORD, and probabilities within the decision tree for having BMD measurement and/or starting treatment were derived for these patients. Only patients who lived in the counties of Uppsala or Östergötland were included in the analyses since data on BMD measurements were available from the single major (DXA responsible) hospitals in these counties. Start cohort was defined for each patient by the first observed event ("index-event”) in SWORD as any of the following: fracture at hip, clinical vertebrae, wrist or other site, glucocorticoid prescription per above criteria, BMD measurement or osteoporosis drug prescription. A wash-out period of two years was used, thus an event was only considered as the index-event if none of the above events had occurred for two years. If the index-event was a fracture, the patient was stratified into one of the fracture start cohorts; if the index-event was glucocorticoid use (filled prescriptions corresponding to $\geq 3$ months at a dose of $5 \mathrm{mg}$ /day of prednisolone, as defined for FRAX) then the start cohort was defined as secondary osteoporosis. If the event was a BMD measurement or osteoporosis treatment, then the start cohort was defined as "other high risk". If the event glucocorticoid use and osteoporosis drug prescription or 
DXA measurement occurred simultaneously then the start cohort was defined as secondary osteoporosis due to glucocorticoid exposure. All events that occurred for a period of two years from the index-event were then tracked and probabilities for having a BMD measurement and/or starting treatment were calculated.

To calculate the number of individuals who have an index-event during one year in Sweden, it was assumed that the population in the regions Uppsala and Östergötland are representative in terms of incidence and demographics for the whole Swedish population.

Probabilities of starting treatment, persistence (in patients who were treated), and switching treatment in patients who were non-persistent over the model simulation were derived from SWORD and stratified by drug. The included pharmaceuticals were chosen in accordance with prescription patterns observed in the research database—alendronate, risedronate, denosumab, zoledronate, teriparatide, strontium ranelate, raloxifene, ibandronate and etidronate.

Probabilities of starting treatment and persistence were measured using Kaplan-Meier survival curves with treatment non-persistence and start, respectively, as failure events. Treatment naïve women and men age $\geq 50$ were identified in SWORD and included for these analyses. Persistence was defined as the number of days from treatment initiation to the end of duration of the last filled prescription of the same treatment or the end of data. Patients were considered persistent if they filled their next prescription of the same drug within 365 days ("permissible gap") after the end of the last prescription. Patients failing to refill their prescription before the end of the permissible gap were defined as non-persistent at the end of the last prescription. Treatment persistence was estimated at 12, 24, 36, 48, and 60 months using Kaplan-Meier survival curves with non-persistence as failure events. Probability of starting treatment over time was measured from two years after index-event and for each year following that point. Patients were censored for death and end of data availability (2012-12-31).

For treatments with a specific treatment length (e.g. teriparatide intended for 18 months' use), patients were assumed to follow the persistence rate observed in the database (although a minority of patients were still on treatment after the intended treatment length) and were assumed non-persistent if treatment was terminated before the intended treatment length. 


\section{Treatment efficacy and duration}

Efficacy data for pharmaceutical treatments were derived from a meta-analysis conducted in an NICE health technology assessment [28]. A 10-year maximum treatment length was assumed as this would reflect that a proportion of patients remain on treatment for longer periods in clinical practice. For example, a Swedish register study showed that, using a 1-year permissible gap between prescriptions, the proportion of patients still persistent to treatment at 5 years after treatment start is about $20 \%$ and it is not unreasonable that a proportion of patients are treated beyond 5 years [6]. Also, safety and effect are documented in 7-10 years in clinical studies [29]. Switching was allowed in the model if discontinuation occurred before maximum treatment length.

\section{Analysis}

\section{Burden of illness}

The model estimates the burden of osteoporosis by employing an incidence-based bottom-up approach containing the number of patients who have an index-event during one year in Sweden multiplied by the corresponding diseaserelated consequences.

Fracture-related costs were presented by incident fractures (direct medical costs incurred in the acute phase of the fracture) and prevalent fractures (fractures that have occurred in previous years but still have an impact on costs and QoL).

To assess the burden inflicted by fractures on quality-adjusted life years (QALYs), an analysis was conducted where QoL and mortality rates in the general population without fractures was compared to the patients who suffered from fractures. Several approaches exist to calculate the intangible cost of lost QALYs, including valuing a QALY lost compared with the general population using an assumed willingness to pay threshold (WTP). Calculating the intangible cost of a lost QALY thus requires an assumption on the value of life as no standardised value exists for the monetary value of a QALY. The WTP for a QALY differs among other things on disease severity, where the society is willing to pay more for patients facing serious conditions. The WTP issued by the Swedish Dental and Pharmaceutical Benefits 
Agency for outpatient drugs was in the interval €79,100-€135,600 during 2005-2011 [30]. A willingness-to-pay threshold of $€ 70,000$ was chosen since it was similar to the lowest cost per QALY in the range. To reach the monetary burden of lost QALYs, the number of lost QALYs was multiplied with the willingness-to-pay threshold.

\section{Scenario analyses}

Given the potential "treatment gap" in Sweden, the current clinical management was compared with five alternative scenarios to assess the impact of making improvements in the management of osteoporosis. The alternative scenarios were chosen to reflect treatment guidelines (described below). The results from cost-effectiveness analyses are presented as the incremental costs and outcomes (QALYs and avoided fractures) for the alternative scenario versus current clinical management. These are presented both per patient and on macro level, i.e. the number of individuals who have an index-event during one year in Sweden multiplied by the per-patient estimated costs and outcomes. The incremental cost-effectiveness ratios (ICERs) were determined by the differences in lifetime total cost divided by the differences in QALYs for the scenario comparison. The cost per QALY are classified in accordance with the Swedish National Board of Health and Welfare guidelines [31]. The monetary investments for achieving the alternative scenarios were calculated as the additional cost in each scenario that could be added until reaching the willingness-topay threshold of €70,000 (henceforth “margin of investment”).

\section{Scenario 1: Increasing persistence by $50 \%$}

Considering the significance of treatment persistence in reducing fracture risk, there is a great potential for improvement regarding improving treatment persistence. In scenario 1, it was assumed that persistence to treatment rates was increased by 50\%, both for patients who started treatment at the beginning of the model simulation and for patients who started treatment at any point after the model start.

\section{Scenario 2: Patients who discontinue treatment switch to next line of treatment}

In scenario 2, it was assumed that patients who discontinue (non-persistence) treatment for any reason switch to next line regardless when the discontinuation occurred. 
Scenario 3: Patients having $\geq 15 \%$ 10-year fracture risk underwent BMD measurement and started treatment if Tscore was $\leq-2$

Swedish national guidelines suggest that patients with a fracture risk according to FRAX of $\geq 15 \%$ (without knowing BMD) should be offered BMD measurement with DXA. If T-score is $\leq-2.0$, then the patients should be offered treatment [32]. It is not known how well this recommendation is followed in clinical practice. In scenario 3, it was assumed this recommendation is fully implemented in clinical management.

\section{Scenario 4: Patients who sustained a hip or vertebral fracture started treatment (disregarding when the fracture occurred)}

The Swedish guidelines also suggest that patients who sustain a hip or vertebral fracture should be offered treatment for osteoporosis [32]. The fourth scenario analysis was therefore conducted where all patients who sustain a hip or vertebral fracture were assumed to start treatment after the fracture, both if it was an index-event or if it occurred during model simulation.

\section{Scenario 5: Scenario 1-4 combined}

To analyse the potential total impact if all the above scenarios were fully implemented, an analysis was conducted by combining the assumptions of scenario 1-4 (scenario 5).

\section{Results}

\section{Patient characteristics}

In total, 19,634 patients were identified in SWORD and included in the analysis. Patient characteristics are shown in Table 1. The most common index-event was an "other" fracture (39\%), followed by wrist fracture (21\%), hip fracture (15\%), glucocorticoid use (13\%), high risk due to other reasons (11\%), and vertebral fracture (2\%). Mean age in all start cohorts was 71 years whilst patients in the hip fracture start cohort were on average older (mean: 80 years) and patients in the wrist and other fracture start cohorts were younger (mean: 68 and 69 years, respectively). The number of patients who have an index-event in Sweden each year was estimated at 54,600. 
[Table 1]

\section{Current clinical management}

Fig. 5 (electronic supplementary material) presents an overview of the simulated current clinical management. A small proportion of patients had BMD measurement following the index-event (13\%). Twenty-six percent started treatment following the index-event, whereof alendronate was the most common treatment (92\% of treatments). Other treatments were evenly distributed. Of patients still alive, 19\%, $14 \%$ and $13 \%$ were on treatment one, five and ten years after index-event, respectively.

The simulated cumulative incidence of any fracture in the current clinical management was estimated at $5 \%, 29 \%$ and $68 \%$ at 1, 5 and 10 years after index-event, respectively (Fig 2). The cumulative incidences of hip, vertebral and wrist fractures were very similar (12\%, 12\% and $11 \%$ respectively at 10 years after index-event) while the incidence of “other” fractures were markedly higher (33\% at 10 years).

[Figure 2]

\section{QALYs lost due to fractures}

QALYs lost due to fractures was estimated at 14,993 over the lifetime of the 54,600 patients with an index-event during one year in Sweden. These were valued at €1,050 million assuming willingness-to-pay per lost QALY of €70,000.

\section{Economic burden}

The total cost was estimated at $€ 776$ million in all patients with an index-event during one year in Sweden. A majority of total cost were attributed to incident fractures (55\%). Costs related to prevalent fractures constituted 36\% while costs of pharmaceuticals and treatment management constituted 3\% and 7\%, respectively. Adding the intangible value of lost QALYs, the total burden was estimated at $€ 1,826$ million. 


\section{Scenario analyses}

The proportion of patients treated for osteoporosis increased in the alternative scenarios 1-5 (Fig. 3). Due to a larger proportion of patients treated, the alternative scenarios prevented a number of fractures (Fig. 4). Table 2 shows the results of the cost-effectiveness analyses in terms of the difference in cost and outcomes of the alternative scenarios as compared with the current clinical management.

[Figure 3]

\section{Scenario 1: Increasing persistence by 50\%}

In scenario 1, the proportion of patients who started treatment at index-event was the same as in the current clinical management; however, those who started treatment in scenario 1 were persistent for a longer time period. Scenario 1 saved in total 403 fractures over 10 years in all patients (0.007/patient) who have an index-event during one year in Sweden, gaining in total 382 QALYs (0.007/patient). Treatment costs increased with $€ 11$ million in the total population. In total, this scenario was cost-saving compared with current clinical management due to decreased fracture-related costs. The "margin of investment” was estimated at €30M (€550/patient) in this scenario.

\section{Scenario 2: Patients who discontinue treatment switch to next line of treatment}

In scenario 2, total cost was higher compared with current clinical management (€14M), while saving 681 QALYs (0.012/patient). The higher total cost is mainly due to a higher proportion of patients treated over time and because most patients switches to more expensive second and third line treatments. Scenario 2 saved 568 total fractures in the population with an index-event during one year in Sweden over 10 years (0.01/patient). The cost/QALY of scenario 2 compared with current clinical management was estimated at €19,783 (moderate cost/QALY). It was estimated that $€ 34 \mathrm{M}$ (€625/patient) could be invested to achieve this scenario.

Scenario 3: Patients having $\geq 15 \%$ 10-year fracture risk underwent BMD measurement and started treatment if Tscore was $\leq-2$

In scenario 3, the proportion of patients who started treatment at index-event was higher compared with current clinical management, while this proportion decreased to a similar level as in the current clinical management over time. In this 
scenario, 1,907 total fractures (0.035/patient) over 10 years were saved. This scenario gained 1,194 total QALYs (0.022/patient). Total cost increased with €38M and the cost/QALY was estimated at €31,368 (moderate cost/QALY). The margin of investment was estimated at $€ 46 \mathrm{M}$ in the total population (€844/patient).

\section{Scenario 4: Patients who sustained a hip or vertebral fracture started treatment (disregarding when the fracture occurred)}

Scenario 4 gained 954 QALYs over the lifetime of the total population, and saved 1,728 fractures in the total population (0.032/patient) during 10 years. The proportion on treatment was similar to scenario 3. However, the estimated QALYs gained were lower in scenario 4 (0.018/patient) compared with scenario 3 (0.022/patient), which reflects that patients with hip/vertebral fracture are on average slightly older than the patients eligible for treatment in scenario 3 . The cost/QALY was estimated at $€ 23,772$, which is classified as moderate.

\section{Scenario 5: Scenario 1-4 combined}

Scenario 5 demonstrates the maximum benefit of the assumed alternative scenarios. Total costs increased with $€ 72 \mathrm{M}$ while gaining 3,864 QALYs. Over 10 years, 4,577 (0.084/patient) fractures were saved. The proportion on treatment over time was markedly higher in scenario 5 compared with scenario 3 and 4 . The cost/QALY in scenario 5 was lower than in scenario 2-4, mainly since patients are assumed persistent to the on average lower-cost first-line treatment as per the assumption in scenario 1.

[Figure 4]

[Table 2]

\section{Discussion}

The objective of this study was to develop a simulation model to assess the burden of disease and cost-effectiveness of different treatment strategies along the osteoporosis treatment pathway. Using Swedish register data this model describes the cost-effectiveness and burden of illness in the real world. Many publications on economic modelling of 
osteoporosis therapies are available, however, to our knowledge this is the first study to analyse the treatment patterns and the cost-effectiveness of osteoporosis management from a real-world healthcare perspective.

QALYs lost in patients who come to clinical attention during one year in Sweden was estimated at almost 15,000 and the healthcare costs in the same population was estimated at $€ 776$ million. These estimates are lower compared with a previous study which used a prevalence-based approach (i.e. examined QALYs and costs related to fractures during a given year regardless of time of disease onset) as opposed to the incident-based approach used in our study [3]. The previous study assessed that osteoporotic fractures caused 39,000 lost QALYs in Sweden during 2010 and the monetary burden of fractures, including cost of incident fractures, prevalent fracture, treatment administration and treatment, in Sweden was estimated at $€ 1,418$ million in 2010 [3]. The estimates are not directly comparable, since two different methodological approaches were used. In this study, we followed incident cases, i.e. who come to clinical attention during one year and accumulate the lifetime costs and outcomes in this group, as compared with the prevalent approach, where the accumulated costs for a population who may have come to clinical attention at any point in time are averaged over a specific year.

The economic cost and individual suffering caused by fractures are high nevertheless, and many studies have concluded that osteoporosis is under-recognised and undertreated in many parts of the world. Sweden has one the highest fracture rates and at the same time the largest gap between those who could be eligible for treatment and those who are treated. The cost-effectiveness analyses in this study indicate that treatment provision could be increased to a low to moderate cost/QALY, or even with potential cost-savings to the society. Although the cost-effectiveness analyses show that these changes may be implemented cost-effectively, the costs related to achieving these improvements in management need to be considered. The cost/investment could be up to €199M and still be considered a cost-effective investment. Intervention programs may be necessary to improve treatment provision which might be associated with increased overhead costs. Such programs can consist of patient education, testing, and more efficient channels to detect patients and follow-up after treatment initiation etcetera. This will be an important issue to address in future studies if data are available. For example, programs such as Fracture Liaison Services (FLS), might be essential to improve management of patients who sustain osteoporotic fractures. Studies have estimated the effectiveness of improving the management of patients sustaining osteoporotic fractures, with varying intervention protocols and results [33, 34]. McLellan et al. 
[35] investigated the cost-effectiveness of FLS in the UK and concluded that FLS is a cost-effective intervention for the prevention of further fractures in fragility-fracture patients (saved $£ 21,000[\sim € 25,000]$ over the lifetimes of 1,000 patients). The same authors conducted a budget-impact analysis of wide-spread adoption of FLS in the UK, which estimated a total set-up cost of establishing 122 additional FLS centres including additional DXA scanners of £9.7M ( €11.6M). This analysis estimated that 31,000 fractures could be prevented over the lifetime of a patients assessed each year. Other studies have shown that FLS are effective in improving diagnosis and treatment of osteoporosis in these patients and cost-effective according to systematic reviews of current evidence [33, 34].

The target population in this study was patients who during a year come to clinical attention. In the research database, we could identify patients who had at least one prescription of osteoporosis drug, and/or typical osteoporotic fracture, glucocorticoid users who also had a prescription of osteoporotic drug and/or fracture, and patients who underwent BMD measurement at the included hospitals. It is possible that some patients who in fact have been at treatment consideration did not appear in the registered data since none of the above events (treatment, fracture, BMD measurement) occurred. Also, it is possible that some individuals did not come to clinical attention but are at high risk. As such, the number of patients who eligible for treatment consideration may be underestimated. However, this issue is considered inconsequential since the scope in this study are patients who have come to clinical attention, and screening for risk patients in the general population is not considered in the current analyses. The number of patients who sustain vertebral fractures is underestimated, as these tend to go undiagnosed [36] and the national patient register only captures fractures diagnosed within specialised care. This should be considered a limitation to this study.

The model can be extended to other analyses. Although the focus in this study was pharmacological treatment, the model can be adapted to assess the impact of non-pharmaceutical interventions, or changes in the management of osteoporosis (e.g. Fracture Liaison Services [37]). It can also be populated with data from other regions or countries, which can provide valuable insights into the burden and how the treatment provision may be improved in different countries. 


\section{References}

1. Johnell, O. and J.A. Kanis, An estimate of the worldwide prevalence and disability associated with osteoporotic fractures. Osteoporos International, 2006(0937-941X (Print)).

2. Hernlund, E., et al., Osteoporosis in the European Union: medical management, epidemiology and economic burden. A report prepared in collaboration with the International Osteoporosis Foundation (IOF) and the European Federation of Pharmaceutical Industry Associations (EFPIA). Arch Osteoporos, 2013. 8: p. 136.

3. Strom, O., et al., Osteoporosis: burden, health care provision and opportunities in the EU: a report prepared in collaboration with the International Osteoporosis Foundation (IOF) and the European Federation of Pharmaceutical Industry Associations (EFPIA). Arch Osteoporos, 2011. 6: p. 59-155.

4. Fleurence, R.L., C.P. Iglesias, and J.M. Johnson, The cost effectiveness of bisphosphonates for the prevention and treatment of osteoporosis: a structured review of the literature.

Pharmacoeconomics, 2007. 25(11): p. 913-33.

5. Jönsson, B., et al., Cost-effectiveness of denosumab for the treatment of postmenopausal osteoporosis. Osteoporosis international, 2011. 22(3): p. 967-982.

6. Jonsson, E., et al., Swedish osteoporosis care. Arch Osteoporos, 2015. 10: p. 222.

7. $\quad$ Solomon, D.H., et al., Osteoporosis medication use after hip fracture in U.S. patients between 2002 and 2011. J Bone Miner Res, 2014. 29(9): p. 1929-37.

8. Landfeldt, E., et al., Adherence to treatment of primary osteoporosis and its association to fractures--the Swedish Adherence Register Analysis (SARA). Osteoporos Int, 2012. 23(2): p. 43343.

9. Strom, O., et al., Cost-effectiveness of alendronate in the treatment of postmenopausal women in 9 European countries--an economic evaluation based on the fracture intervention trial. Osteoporos Int, 2007. 18(8): p. 1047-61.

10. Borgstrom, F., et al., An economic evaluation of strontium ranelate in the treatment of osteoporosis in a Swedish setting: based on the results of the SOTI and TROPOS trials. Osteoporos Int, 2006. 17(12): p. 1781-93.

11. Kanis, J.A., et al., Long-term risk of osteoporotic fracture in Malmo. Osteoporos Int, 2000. 11(8): p. 669-74.

12. Kanis, J.A., et al., The burden of osteoporotic fractures: a method for setting intervention thresholds. Osteoporos Int, 2001. 12(5): p. 417-27.

13. Statistics Sweden, Ettårig livslängdstabell, dödsrisker (promille) efter kön, ålder och år [One year life expectancy, mortality (per mille) by sex, age and year]. 2015, Statistics Sweden: http://www.statistikdatabasen.scb.se/sq/20061.

14. Strom, O., B. Jonsson, and J.A. Kanis, Intervention thresholds for denosumab in the UK using a FRAX(R)-based cost-effectiveness analysis. Osteoporos Int, 2013. 24(4): p. 1491-502.

15. Kanis, J.A., et al., Excess mortality after hospitalisation for vertebral fracture. Osteoporos Int, 2004. 15(2): p. 108-12.

16. Bliuc, D., et al., Mortality risk associated with low-trauma osteoporotic fracture and subsequent fracture in men and women. JAMA, 2009. 301(5): p. 513-21.

17. Kanis, J.A., et al., The components of excess mortality after hip fracture. Bone, 2003. 32(5): p. 468-73.

18. von Friesendorff, M., et al., Hip fracture, mortality risk, and cause of death over two decades. Osteoporos Int, 2016. 27(10): p. 2945-53.

19. Tandvårds- och Läkemedelsförmånsverket (The Dental and Pharmaceutical Benefits Agency), Läkemedelsförmånsnämndens allmänna råd om ekonomiska utvärderingar in TLVAR 2017:1, T.o. Läkemedelsförmånsverket, Editor. 2017. 
20. Borgstrom, F., et al., Costs and quality of life associated with osteoporosis-related fractures in Sweden. Osteoporos Int, 2006. 17(5): p. 637-50.

21. The Dental and Pharmaceutical Benefits Agency (TLV), Läkemedelsdatabas. 2016, The Dental and Pharmaceutical Benefits Agency.

22. Södra Regionvårdsnämnden, Regionala priser och ersättningar för södra sjukvårdsregionen 2016. 2015.

23. Statistics Sweden, Consumer price index $(1980=100)$ [Online database]. 2016: http://www.scb.se/hitta-statistik/statistik-efter-amne/priser-ochkonsumtion/konsumentprisindex/konsumentprisindex-kpi/.

24. The Riksbank, Search interest \& exchange rate [Online Database], Riksbanken (Sweden's central bank), Editor. 2016: http://www.riksbank.se/en/Interest-and-exchange-rates/search-interest-ratesexchange-rates/.

25. ICUROS investigators, Quality of Life after Osteoporotic Fractures - Results from the ICUROS [Unpublished data]. 2016.

26. Zethraeus, N., et al., Costs and Quality of life Associated with Osteoporosis Related Fractures Results from a Swedish Survey, N. SSE/EFI Working Paper Series in Economics and Finance, Editor. 2002.

27. Burstrom, K., M. Johannesson, and F. Diderichsen, Swedish population health-related quality of life results using the EQ-5D. Qual Life Res, 2001. 10(7): p. 621-35.

28. National Institute for Health and Care Excellence (NICE), Alendronate, etidronate, risedronate, raloxifene and strontium ranelate for the primary prevention of osteoporotic fragility fractures in postmenopausal women. 2008.

29. Läkemedelsverket (Medical Products Agency), Behandling av osteoporos för att förebygga frakturer - Behandlingsrekommendation. 2004.

30. Svensson, M., F.O. Nilsson, and K. Arnberg, Reimbursement Decisions for Pharmaceuticals in Sweden: The Impact of Disease Severity and Cost Effectiveness. Pharmacoeconomics, 2015. 33(11): p. 1229-36.

31. Socialstyrelsen (National Board of Health and Welfare), Nationella riktlinjer för sjukdomsförebyggande metoder 2011 Hälsoekonomiskt underlag [National guidelines for disease prevention methods 2011]. 2011.

32. Socialstyrelsen (National Board of Health and Welfare), Nationella riktlinjer för rörelseorganens sjukdomar 2012. 2012.

33. Marsh, D., et al., Coordinator-based systems for secondary prevention in fragility fracture patients. Osteoporos Int, 2011. 22(7): p. 2051-65.

34. Sale, J.E., et al., Systematic review on interventions to improve osteoporosis investigation and treatment in fragility fracture patients. Osteoporos Int, 2011. 22(7): p. 2067-82.

35. McLellan, A.R., et al., Fracture liaison services for the evaluation and management of patients with osteoporotic fracture: a cost-effectiveness evaluation based on data collected over 8 years of service provision. Osteoporos Int, 2011. 22(7): p. 2083-98.

36. Cooper, C., et al., Incidence of clinically diagnosed vertebral fractures: a population-based study in Rochester, Minnesota, 1985-1989. J Bone Miner Res, 1992. 7(2): p. 221-7.

37. Javaid, M.K., et al., Effective secondary fracture prevention: implementation of a global benchmarking of clinical quality using the IOF Capture the Fracture $(R)$ Best Practice Framework tool. Osteoporos Int, 2015. 26(11): p. 2573-8. 


\section{Figure legends}

Fig. 1: Model structure. Patients start to the left in the decision tree and transits to the Markov model after treatment decision

Fig. 2: Simulated cumulative incidence of fractures by year after index-event in the current clinical management

Fig. 3: Proportion on treatment in each alternative scenario (1-5) and current clinical management

Fig. 4: Number of avoided fractures by scenario compared with current clinical management during 10 years, in all patients who have an index-event in one year in Sweden

\section{Electronic supplementary material}

Fig. 5: Simulated current clinical management from index-event. Each point in the graph, from right to left, shows the proportion of patients by each start-cohort (“index-event”). From the index-event, patients to the decision tree and undergo BMD measurement or not, moves to treatment decision, and the projected treatment status at year 1,5 and 10 after index-event. The width of the lines indicates the proportion of patients within each state. 


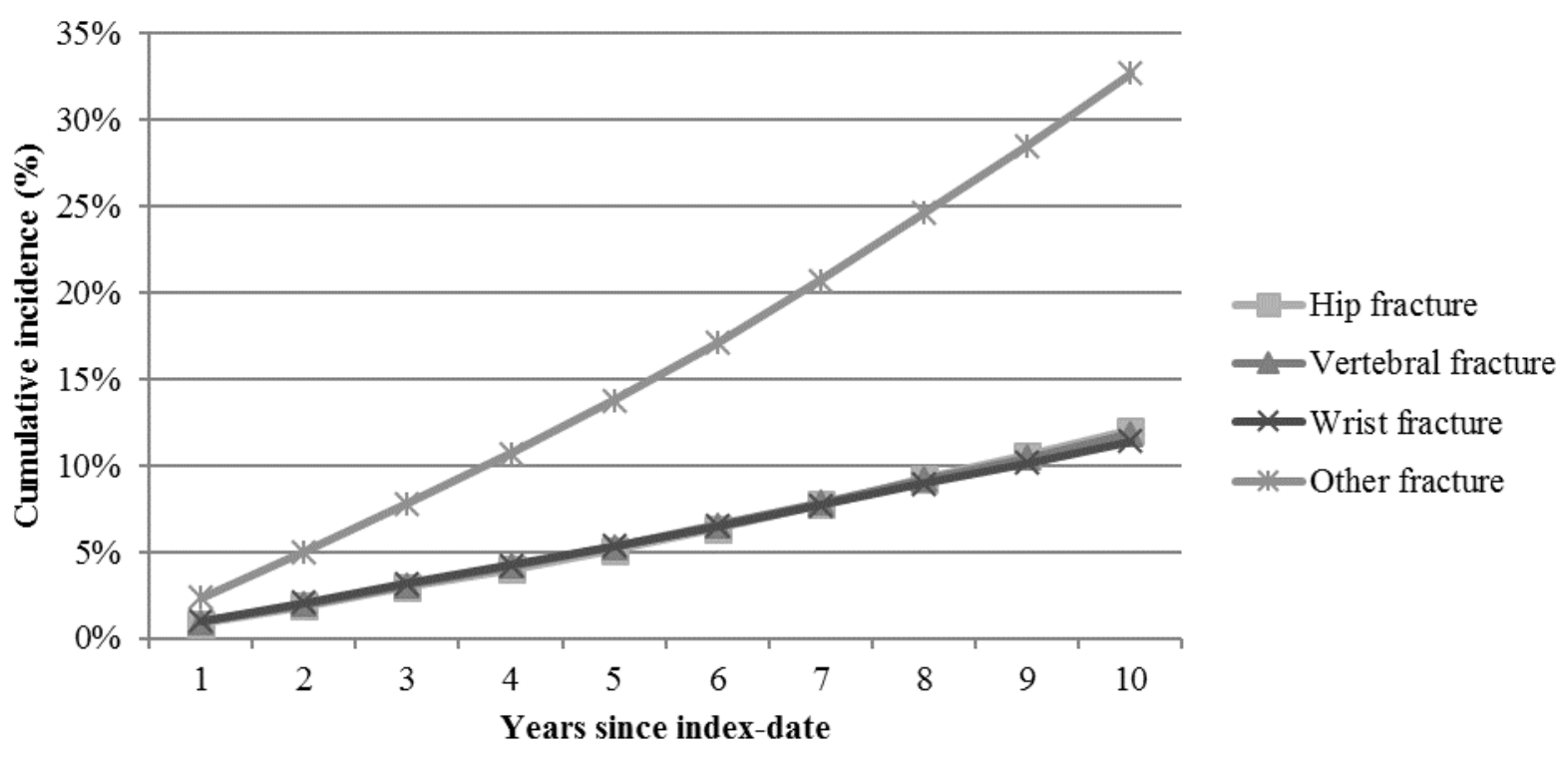


Table 1. Patient characteristics

\begin{tabular}{llll}
\hline Start cohort & $\mathbf{N}(\%)$ & Age, mean years (SD) & Female, n (\%) \\
\hline All cohorts & $19,634(100)$ & $71.3(11.9)$ & $13,810(70.3)$ \\
Hip fracture & $2,827(14.4)$ & $80.1(10.1)$ & $1,949(68.9)$ \\
Vertebral fracture & $339(1.7)$ & $78.2(9.9)$ & $239(70.5)$ \\
Wrist fracture & $4,101(20.9)$ & $67.9(11.2)$ & $3,339(81.4)$ \\
Other fracture & $7,586(38.6)$ & $68.7(12.0)$ & $4,789(63.1)$ \\
Glucocorticoid use & $2,785(14.2)$ & $71.8(10.2)$ & $1,828(65.6)$ \\
Other high risk & $1,996(10.2)$ & $74.2(9.8)$ & $1,666(83.5)$ \\
\hline
\end{tabular}


Table 2. Results from the cost-effectiveness analyses, presented as incremental costs and outcomes in the alternative scenario vs. current clinical management (per patient/total population)

\begin{tabular}{|c|c|c|c|c|c|}
\hline & $\begin{array}{c}\text { Scenario 1: } \\
\text { increasing } \\
\text { persistence by } \\
50 \% \\
\text { vs. current } \\
\text { pathway } \\
\end{array}$ & $\begin{array}{l}\text { Scenario 2: } \\
\text { non-persistent } \\
\text { patients switch } \\
\text { to next line of } \\
\text { treatment } \\
\text { vs. current } \\
\text { pathway } \\
\end{array}$ & $\begin{array}{l}\text { Scenario 3: patients with } \\
\geq 15 \% 10 \text {-year fracture } \\
\text { risk (FRAX) undergo } \\
\text { BMD measurement and } \\
\text { start treatment if } T \text { - } \\
\text { score } \leq-2.0\end{array}$ & $\begin{array}{c}\text { Scenario 4: } \\
\text { patients who } \\
\text { sustain a } \\
\text { hip/vertebral } \\
\text { fracture start } \\
\text { treatment } \\
\text { vs. current } \\
\text { pathway } \\
\end{array}$ & $\begin{array}{l}\text { Scenario 5: } \\
\text { Scenario 1- } \\
4 \text { combined }\end{array}$ \\
\hline \multicolumn{6}{|l|}{ Costs } \\
\hline $\begin{array}{l}\text { Treatment cost } \\
\text { difference }\end{array}$ & $\begin{array}{c}€ 193 / \\
€ 10.6 \mathrm{M}\end{array}$ & $\begin{array}{c}€ 651 / \\
€ 35.6 \mathrm{M}\end{array}$ & $\begin{array}{l}€ 1,306 / \\
€ 71.4 \mathrm{M}\end{array}$ & $\begin{array}{l}€ 1,283 / \\
€ 70.1 \mathrm{M}\end{array}$ & $\begin{array}{l}€ 3,775 / \\
€ 205.2 \mathrm{M}\end{array}$ \\
\hline $\begin{array}{l}\text { Cost of incident } \\
\text { fractures } \\
\text { difference }\end{array}$ & $\begin{array}{l}€-152 \text { / } \\
€-8.3 M\end{array}$ & $\begin{array}{c}€-253 / \\
€-13.8 M\end{array}$ & $\begin{array}{c}€-363 / \\
€-19.8 M\end{array}$ & $\begin{array}{c}€-603 / \\
€-33.0 \mathrm{M}\end{array}$ & $\begin{array}{l}€-1,512 / \\
€-82.6 \mathrm{M}\end{array}$ \\
\hline $\begin{array}{l}\text { Cost of } \\
\text { prevalent } \\
\text { fractures } \\
\text { difference }\end{array}$ & $\begin{array}{l}€-101 / \\
€-5.5 \mathrm{M}\end{array}$ & $\begin{array}{l}€-152 \text { / } \\
€-8.3 \mathrm{M}\end{array}$ & $\begin{array}{c}€-258 / \\
€-14.1 \mathrm{M}\end{array}$ & $\begin{array}{c}€-265 / \\
€-14.5 \mathrm{M}\end{array}$ & $\begin{array}{c}€-928 / \\
€-50.7 \mathrm{M}\end{array}$ \\
\hline $\begin{array}{l}\text { Total cost } \\
\text { difference }\end{array}$ & $\begin{array}{l}€-60 / \\
€-3.3 \mathrm{M}\end{array}$ & $\begin{array}{c}€ 247 / \\
€ 13.5 \mathrm{M}\end{array}$ & $\begin{array}{c}€ 686 / \\
€ 37.5 \mathrm{M}\end{array}$ & $\begin{array}{c}€ 517 / \\
€ 28.3 \mathrm{M}\end{array}$ & $\begin{array}{l}€ 1,315 \text { / } \\
€ 71.9 \mathrm{M}\end{array}$ \\
\hline QALYs gained & $\begin{array}{c}0.007 / \\
382\end{array}$ & $\begin{array}{c}0.012 / \\
681\end{array}$ & $\begin{array}{c}0.022 / \\
1,194\end{array}$ & $\begin{array}{c}0.018 / \\
954\end{array}$ & $\begin{array}{c}0.071 / \\
3,864\end{array}$ \\
\hline $\begin{array}{l}\text { Incremental } \\
\text { cost- } \\
\text { effectiveness } \\
\text { ratio (ICER) }\end{array}$ & Cost-saving & $€ 19,783$ & $€ 31,368$ & $€ 23,772$ & $€ 18,605$ \\
\hline $\begin{array}{r}\text { Margin of } \\
\text { investment }\end{array}$ & $\begin{array}{c}€ 550 / \\
€ 30.1 \mathrm{M} \\
\end{array}$ & $\begin{array}{c}€ 626 / \\
€ 34.2 \mathrm{M} \\
\end{array}$ & $\begin{array}{c}€ 844 / \\
€ 46.1 \mathrm{M} \\
\end{array}$ & $\begin{array}{c}€ 807 / \\
€ 44.1 \mathrm{M} \\
\end{array}$ & $\begin{array}{l}€ 3,634 / \\
€ 198.6 \mathrm{M} \\
\end{array}$ \\
\hline
\end{tabular}




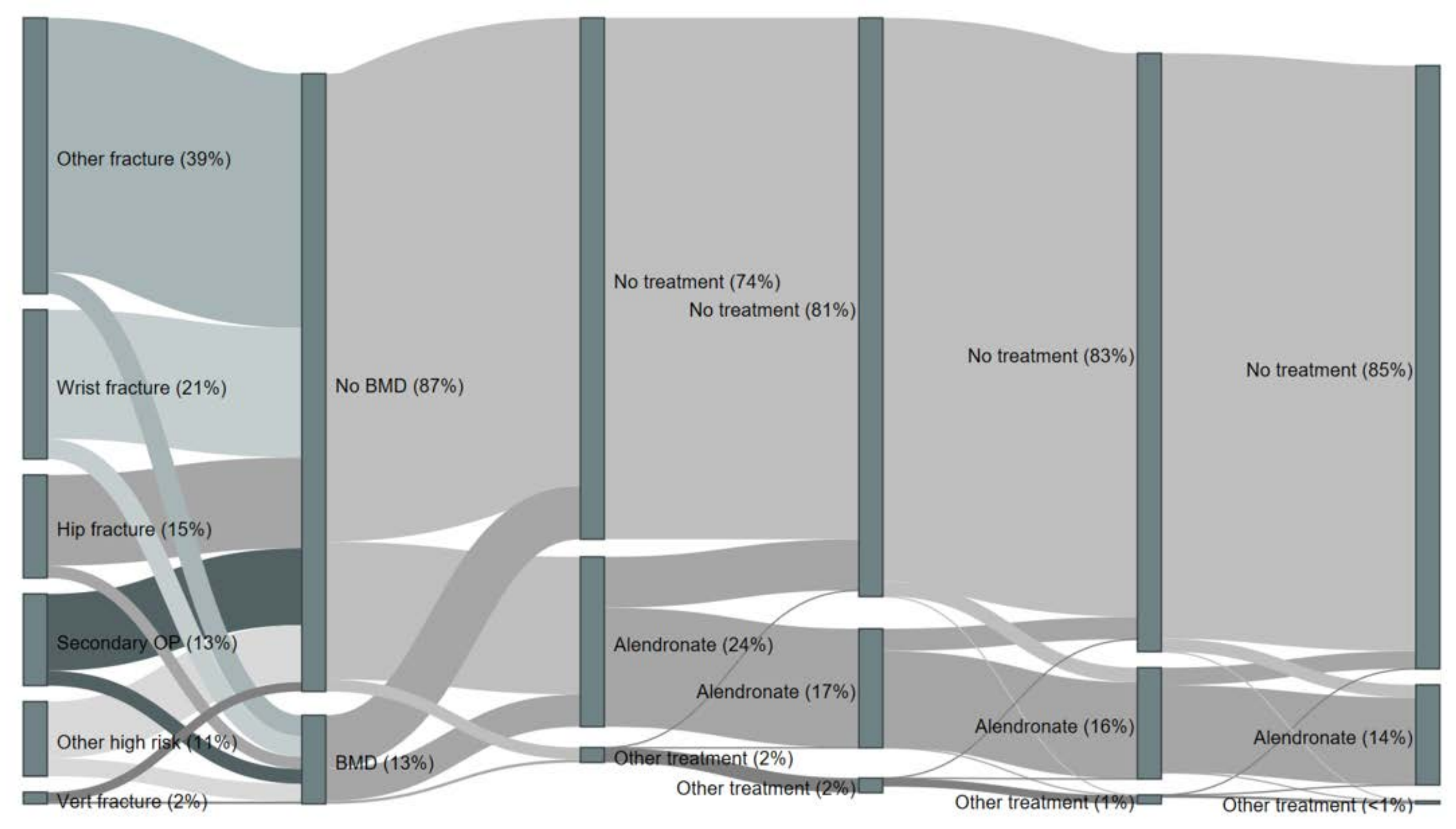

